\title{
SHORT-LIVED DECAY PRODUCTS OF RADON-222 AND RADON-220 \\ IN AIR AT HACHIJO-JIMA, CHICHI-JIMA AND NAGOYA : APPLICATION FOR DETERMINING AEROSOL RESIDENCE TIME
}

\author{
M. Shimo, Y. Ikebe, and H. Ogawa \\ Department of Nuclear Engineering \\ Faculty of Engineering, Nagoya University \\ Nagoya 464
}

\begin{abstract}
Concentrations of radon-222 short-lived decay products were measured in surface air on the windward sides of Hachijo-jima and Chichi$j$ ima and on the campus of Nagoya University. Concentrations of radon-222 gases and thoron short-lived decay products were also obtained simultaneously on the same site at Hachijo-jima. On the basis of the radon concentration data and meteorological trajectories, decay time constants for tropospheric aerosols due to dilution and mean residence times for atmospheric aerosol particles over ocean areas are estimated to be $0.48 \sim$ 2.5 days and $0.13 \sim 0.67$ days, respectively.
\end{abstract}

\section{Introduction}

Radon-222(Rn), a naturally occuring chemically inert radioactive gas, is produced by the decay of Ra-226 in soils. The Rn exhalation rate over continents is approximately 100 times that over oceans. The only significant mechanism for removing $\mathrm{Rn}$ from atmosphere is by radioactive decay( 3.82 days half life). These facts make $R$ an ideal tracer for large seale transport of air masses across oceans.

In recent years, the decay time constant for lower atmospheric aerosols due to dilution has been estimated from the concentrations of short-lived decay products of radon(Rn-Dts) which has been assumed to be in equilibrium state with Rn (Misaki et al.,1975, Mochizuki and Tanji, 1981). Misaki et al. presented to be about 2.5 days for the decay time constant for aerosol particles due to dilution and Mochizuki and Tanji indicated the values ranging from 1.3 to 2.7 days.

The aerosol residence time in the lower atmospheric air has been estimated from the rate of the concentration of long-lived radioactive nuclides (e.g. Poet et al., 1972, Moore et al., 1979, and others), and from the concentration of aerosol particles (Morita et al.,1973). A number of independent estimations based on $\mathrm{Po}-210 / \mathrm{Pb}-210$ ratio indicated fairly long tropospheric aerosol residence time of almost one month. However, Poet et al. represented an apparent aerosol residence time averaging only a few days or less. Tsunogai(1980) presented to be 10 l4 days for aerosol residence times on the basis of $\mathrm{Bi}-210 / \mathrm{Pb}-210$ and $\mathrm{Po}-210 / \mathrm{Pb}-210$ ratios. On the other hand, from the results of observations of aerosol particles, Misaki et al. estimated apparent aerosol residence times to be 0.35 days (for particle radius $0.5 \mu \mathrm{m}$ ) and 5 days (for particle radius $0.03 \mu \mathrm{m}$ ), and Morita et al. obtained those ranging from 0.15 to 0.63 days.

The purpose of the present study is to estimate the decay time constant for lower atmospheric aerosols due to dilution $\left(\tau_{d}\right)$ and the 
aerosol residence time $\left(\tau_{r}\right)$ on the basis of the data observed - ( 1 ) concentration of Rn-Dts in surface air carried from the Japan Islands to Hachijojima and Chichi-jima, (2) the radioactive equilibrium state between $R_{n}$ and Rn-Dts in surface air on Hachijo-jima, and (3) Tn-Dts concentration in surface air on the two islands.

\section{Measurements}

The Rn-Dts were collected on a $1.2 \mu \mathrm{m}$ pore size filter (Millipore

Filter RA) ; this filter was nearly 100 percent efficient and also had very little self absorption during counting. Sampling times were $60 \mathrm{~min}, 30 \mathrm{~min}$, and $15 \mathrm{~min}$ at almost $10 \mathrm{l} / \mathrm{min}, 50 \mathrm{l} / \mathrm{min}$, and $20 \mathrm{l} / \mathrm{min}$, at Hachijo-jima, Chichi-jima, and Nagoya, respectively. The activities on the sampled filter counted with zinc-sulfide alpha scintillation counting system. The instruments used at Chichi-jima and Nagoya were operated automatically except once exchange of filter paper a day.

The Rn concentration was measured by the use of a continuous flowtype ionization chamber. A sample air, passed through a filter to eliminate Rn- and Tn-Dts, was admitted to the ionization chamber at almost $1.0 \mathrm{l} / \mathrm{min}$. Ionization current was measured by a vibrating-reed-electrometer ; the electric signals caused by alpha particles were selected from those by beta-, gamma, and cosmic rays using an advanced pulse technique. This technique will be described in detail in the near future.

The measurement of Tn-Dts was carried out with manual operation using the filter-pack technique applied for Rn-Dts measurement. The sampling time was about one day and the counting time to obtain a decay curve was several hours.

The measurements of $\mathrm{Rn}$ - and Tn-Dts concentrations were carried out from January 14 to 20, 1981, on the two islands (Hachijo-jima and Chichijima) and the measurement of Rn-Dts concentration at the campus of Nagoya University. In addition to these measurements, Rn concentration was also obtained at Hachijo-jima.

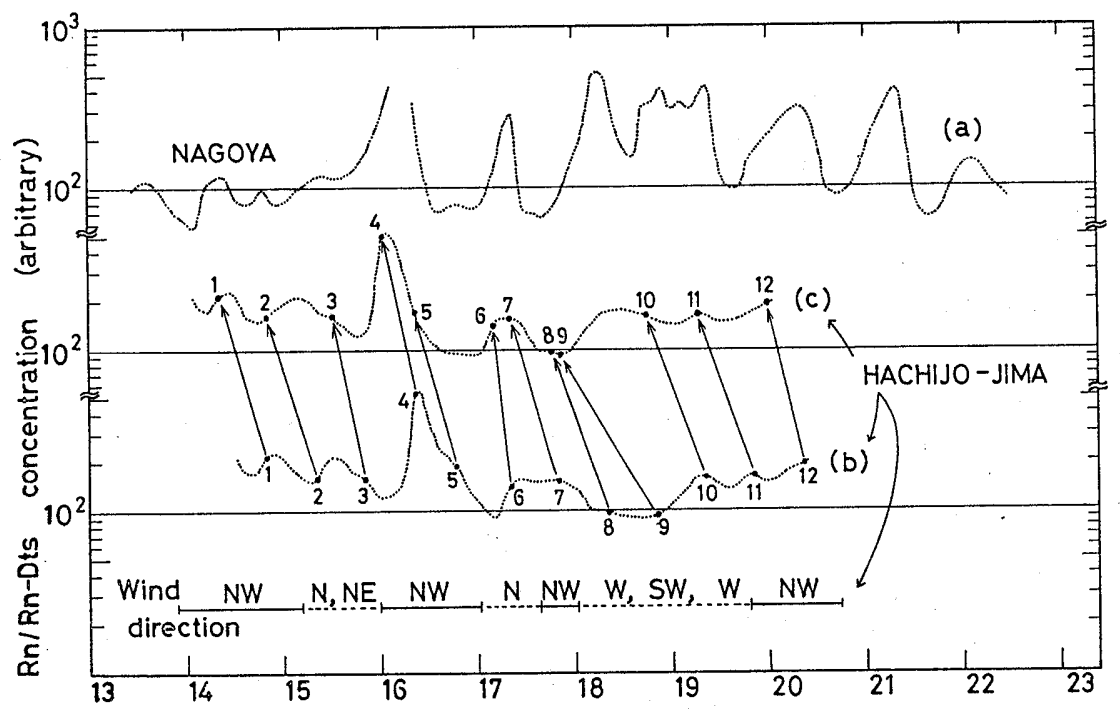

Jan. 1981

Fig. 1. Diurnal variations of $R n$ and Rn-Dts concentration. 


\section{Results and Discussions}

Figure I shows duirnal variations of Rn-Dts concentration measured at Nagoya (a) and of Rn concentration on Hachijo-jima (b). On the basis of air masses trajectories, transit times of air masses from the Japan Islands to Hachijo-jima were estimated to be $8 \sim 12$ hours during observation period. The curve (c) indicates a correction curve; this is shifted to the left direction on time axis by only transit time from the curve (b). Radioactive decay during transit was neglected since the half life of $\mathrm{Rn}$ is 3.82 days. Not only the concentration but also a pattern of variation shown by the curve (c) agreed fairly well with those shown by the curve (a).

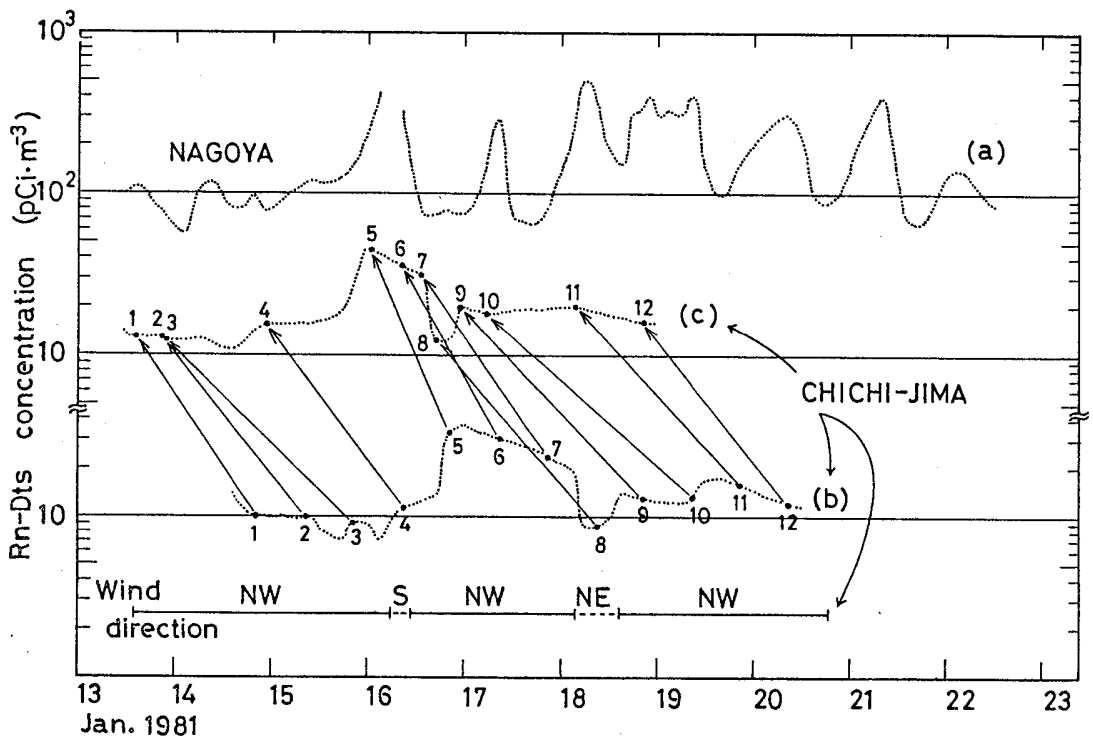

Fig. 2. Diurnal variation of Rn-Dts concentration.

Figure 2 shows the result observed on Chichi-jima ; the curve (c) involved correction based on transit times $(1 \sim 1.5$ days) in the same manner as Fig.l(c). In this case, the correction of radioactive decay of $\mathrm{R}_{n}$ during transit was also made. The Rn-Dts concentration of Chichi-jima was almost one-tenth of Nagoya. It was found that the temporary variation of Rn-Dts of Chichi-jima agreed roughly that of Nagoya, although the variation of Chichi-jima was rather blunt.

Figure 3 shows examples of the decay of radioactivities sampled on filters, and indicates that Tn-Dts was able to be detected in surface air on Hachijo-jima but was not detected on Chichi-jima.

Figure 4 shows the good correlation between Rn and Rn-Dts in air on Hachijo-jima and a close agreement of concentration between them suggests the radioactive equilibrium state. Since $R_{n}$ is in a gaseous state in air, it dissipates from air mass due to radioactive decay and dilution process. On the other hand, Rn-Dts vanish due to the radioactive decay and the scavenging and removal processes as aerosol partivles. Nevertheless, the radioactive equilibrium state between $R_{n}$ and $R_{n}-D t s$ is presumed in air over oceans since the half life of $\mathrm{Rn}_{\mathrm{n}}$ is considerably long compared with that of $\mathrm{R}_{n}-\mathrm{Dts}(0.0278$ days) and the $\tau_{d}$ is larger than the radioactive half life of Rn-Dts. 

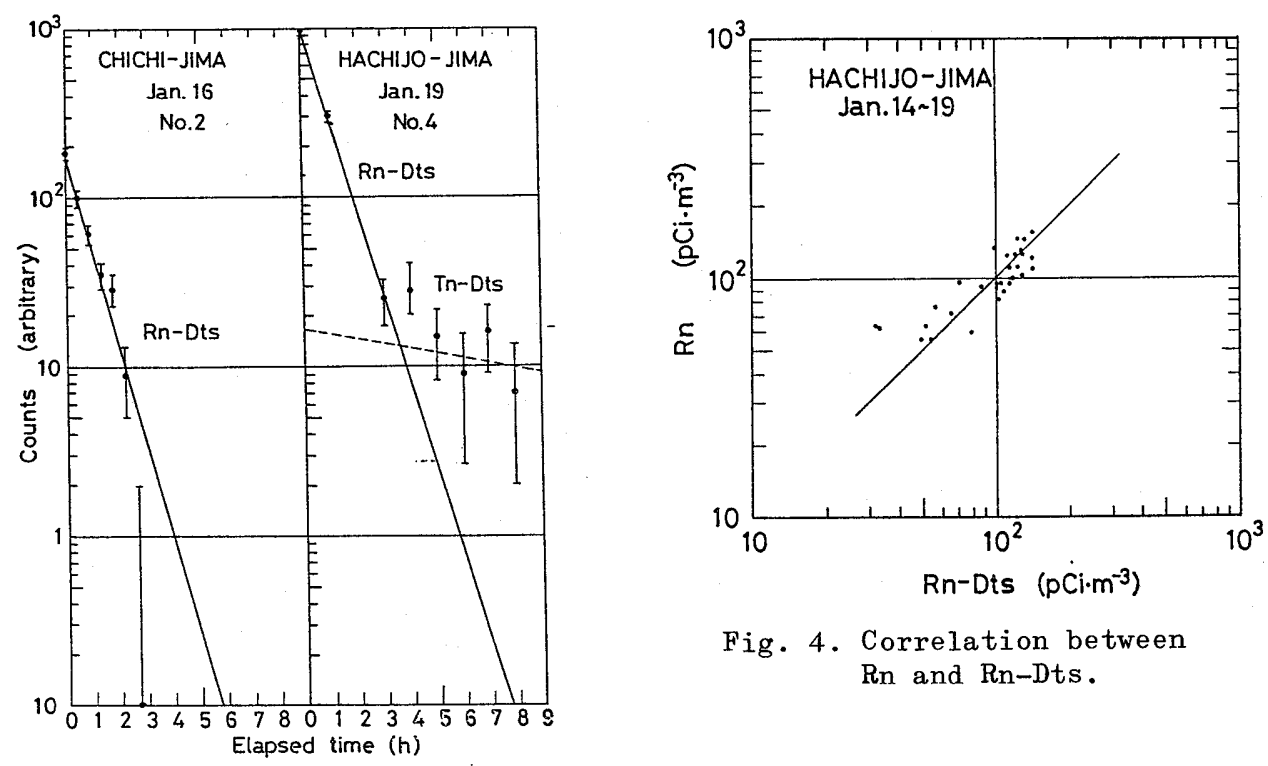

Fig. 4. Correlation between $\mathrm{Rn}$ and $\mathrm{Rn}-\mathrm{Dts}$.

Fig. 3. Decay curve of radioactivity.

Figure 5 shows the variation of mean concentrations of $R_{n}$ and/or Rn-Dts and Tn-Dts as a function of distance from the Japan Islands. The degree of the decrease of Tn-Dts concentration is more acute than that

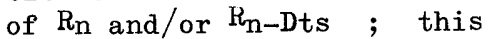
is because (1) Tn-Dts does not accompany with its parent nuclide ( $\mathrm{T} n$ ) whose half life is 54 seconds, (2) the half life of Tn-Dts (ThB : 10.6 hours) is short compared to that of $R_{n}$, and (3) Tn-Dts are considered to exist as radioactive aerosol particles in atmosphric air.

Let $Q$ and $Q_{0}$ represent radioactivity concentration on the island (Hachijo-jima and/or Chichi-jima) and the Japan Islands, respectively, we have

$$
Q=Q_{0} \exp (-\wedge t)
$$

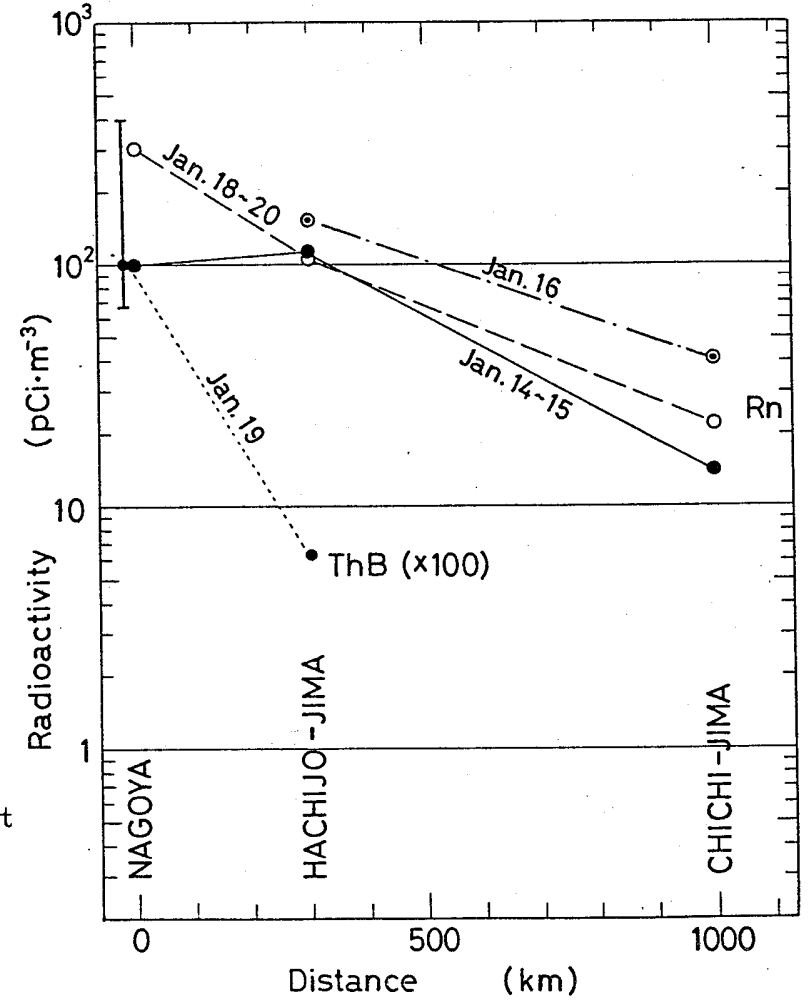

Fig. 5. Radioactivity vs. distance. 
where $\Lambda$ is an apparent removal rate for $\mathrm{Rn}$ and/or $\mathrm{Tn}-\mathrm{Dts}$ and $t$ denotes the time length taken after ${ }_{R}$ the air mass left the Japan Islands. The apparent removal rate for $\mathrm{Rn}, \Lambda^{\mathrm{R}}$, is expressed as follows

$$
\Lambda^{R}=\lambda_{a}^{R}+\lambda_{d}
$$

where $\lambda^{R}$ is the radioactive decay constant of $R n\left(0.182 d^{-1}\right)$ and $\lambda_{d}$ is the removal ${ }^{a}$ rate for atmospheric particles due to dilution process.

In the case of Tn-Dts, the apparent removal rate of $\mathrm{Tn}-\mathrm{Dts}, \Lambda^{\mathrm{T}}$, is represented as follows

$$
\Lambda^{\mathrm{T}}=\lambda_{\mathrm{a}}^{\mathrm{T}}+\lambda_{\mathrm{d}}+\lambda_{\mathrm{r}}
$$

where $\lambda_{a}^{\mathrm{T}}$ is the radioactive decay constant of $\operatorname{Tn}-\operatorname{Dts}\left(1.57 \mathrm{~d}^{-1}\right)$ and $\lambda$ is the removal rate for atmospheric particles due to the combined action of all particle scavenging and removal processes excep $\ddagger$ dilution process.

The $\lambda_{\mathrm{d}}$ was estimated to be $0.48 \sim 2.5 \mathrm{~d}^{-1}\left(\tau_{\mathrm{d}}=1 / \lambda_{\mathrm{d}}=1.2\right.$ days; averaged individual $\lambda_{d}$ ) from Eq.s. (1) and (2) usjng the data shown in Fig.5 and mean wind vefocity. On the other hand, the $\Lambda$ were estimated to be $2.5 \sim$ $8.5 \mathrm{~d}^{-1}\left(\tau=1 / \Lambda^{\mathrm{T}}=0.12 \sim 0.4\right.$ days $)$ from the data shown in Fig. 5. Hence, the $\lambda$ was found from cafculation to be $1.5 \sim 7.5 \mathrm{~d}^{-1}\left(\tau_{\mathrm{r}}=1 / \lambda_{\mathrm{r}}=0.67 \sim 0.13\right.$

The result estimated almost agrees with the value obtained by Morita et al. and roughly agrees with that by Misaki et al. However, there are disagreement between the present work and other researchers (Poet et al. and Tsunogai). These discrepancy shall be discussed after the appointed observation during 1981 1983, taking account of : (1) local anomaly, (2) vertical profile, (3) diurnal variation, and (4) seasonal variation for the concentrations of Rn, its long-lived decay products, and Tn-Dts.

Acknowledgments .

Mr. T. Itoh had the Kindness to provide us the air mass trajectory maps. The authors wish to express their thanks to him for his help.

\section{References}

Misaki, M., M. Ikegami, and I. Kanasawa, Deformation of the size distribution of aerosol particles dispersing from land to ocean, J. Met. Soc. Japan 53, 111-120, 1975.

Mochizuki, S. and T. Tanji, Radioactive aerosols in the atmosphere over ocean near Japan Islands (in Japanese), Res. Lett. Atmosph. Electr., $\underline{1}$,

Poet, S. E., H. E. Moore, and E. A. Martell, Lead 210, Bismuth 210, and polonium 210 in the atmosphere : Accurate ratio measurement and application to aerosol residence time determination, J. Geophys. Res., 77, 6515 $-6527,1972$.

Moore, H. E., S. E. Poet, and E. A. Martell, Vertical profiles of Rn-222 and its long-lived daughters over the Eastern Pacific, Environ. Sci. Techn., 11, 1207-1210, 1977.

Morita, Y., H. Ishikawa, J. Nagasaka, and M. Kanada, The land-to-ocean transitional behavior of the atmospheric electric parameters and their relation to atmospheric pollution, J. Met. Soc. Japan, 5l, 294-306, 1973. Tsunogai, S., Report of Special Research Projects on Environmental Science,
1980.

(Received February 1, 1981; revised March 3, 1982; accepted March 31, 1982) 